\title{
Big lessons about small things
}

\author{
Teaching a diverse field such as nanotechnology is far from easy. Doug Natelson provides a few pointers.
}

A $s$ readers of this journal will know, nanotechnology is an extremely broad field, which cuts across many traditional disciplines, from condensedmatter physics to biology from quantum chemistry to electrical engineering. Given this breadth, and the highly technical nature of the topic, determining the best pedagogical approaches to take is a considerable challenge. At Rice University, we have been thinking about nanoscience education for the better part of two decades, and I have been teaching broad-based, first-year graduate level courses on the subject since 2002. I have some insight into the process and I provide here a few key points. These are, of course, only my opinions and reasonable people may disagree with some or all of them. Be forewarned: my views are from the physics perspective, although my background experience in engineering colours them as well.

Nanotechnology is so broad that it is extremely difficult to cover well within a single, traditional academic department, particularly for an undergraduate course of study. I know that nanotechnology departments and degree programmes exist, involving high-quality faculty, but teaching a programme with such enormous breadth seems to me like it would be an uphill struggle. Majoring in nanotechnology could be like majoring in 'engineering'. Trying to educate an undergraduate broadly in nanotechnology or nanoscience runs the risk of producing a student who knows a little physics, a little chemistry or a little materials science, but would not have mastery in any one area. We teach a two-semester nanophysics course sequence that reviews solid-state physics from the nano perspective, and discusses the nano side of electronic devices, magnetism, photonics, micro/ nanomechanical systems, micro/nanofluidics and touches on the nano/bio interface. To get an in-depth technical grounding in all of this, to say nothing of the chemistry and bio aspects of nano and the relevant characterization and fabrication techniques, would be very challenging. Constructing and teaching a curriculum encompassing this breadth of material at an accessible level would require a deep investment of instructional effort and resources. Teaching a shallow, hand-wavy version of nano, without the quantitative technical depth, would do more harm than good, in my view.

If you want to be a nano expert, first become an expert in a traditional discipline. $\mathrm{A}$ major part of the undergraduate educational experience is learning what it really means to know large portions of the relevant disciplines in depth, and in my view this is prerequisite to becoming adept in an interdisciplinary field.

Teach the macro so that people appreciate why the nano is different. In some sense, the nanoscale is where many approximations tacitly made in traditional undergraduate courses go to die. Students need to understand what a bandgap is before they can appreciate quantum confinement in semiconductor nanocrystals. Students need to understand how the symmetry of a crystal lattice leads to crystal momentum conservation before they can appreciate how nanocrystals can help evade that constraint in optical processes. Students need to know about the idea of a continuum fluid with a density and a viscosity to appreciate why nanoconfined fluids can behave very differently. I've found that one way to maintain student interest is to point out that some key concept they were taught as undergraduates doesn't really work at the nanoscale, such as the idea of a friction coefficient.

\section{Emphasize the fascinating commonalities} that run through nanoscience. Some themes crop up repeatedly in physics in general and nanoscience in particular, and such threads can help guide students' intuitions. Hybridization is one such idea: couple together a bunch of identical oscillators, and you end up with a band of new normal modes, with a spread in frequencies related to the strength of the coupling. This is the same basic concept behind the formation of energy bands as atomic wavefunctions are made to overlap, the formation of minibands as semiconductor quantum wells are coupled via the tails of the well bound states, the formation of photonic bands in periodic dielectric structures, and so on. Another example is the importance of boundary conditions: it's the imposition of boundary conditions that takes systems with continuous (energy/frequency) spectra and picks out a discrete spectrum. This happens for the wavefunction of a particle-in-a-box; for the electromagnetic field in optical cavities; for the (envelope) electronic wavefunction in quantum wells and quantum dots; for plasmons confined to nanoparticles; for the displacement of a mechanical resonator, even a macroscale guitar string. Pointing out recurring motifs helps students tie complex ideas together.

Quantum really is critically important. Some popular treatments of nanoscience try to downplay the importance of quantum mechanics; others spend too much time trying to play up the 'weirdness' of the quantum regime. There's no question that a solid grounding in quantum concepts (wavefunctions, tunnelling, densities of states, rates of processes and the origins of selection rules) is extremely helpful as baseline knowledge for any aspiring nano student.

Teach fundamentals, because they remain fundamental. Trying to teach the state-ofthe-art is incredibly difficult because it is a continuously moving target. However, the basic physical and chemical concepts behind nanoscience are well established and are still going to be scientifically valid throughout the careers of your students. If your students have an appreciation, understanding and intuition based on those fundamentals, they will be positioned to adapt to the latest trends, whether they go into industry or academia.

Now is the time to take stock. Nanoscience and nanotechnology have reached a level of maturity where we can develop some sense of perspective about education in the area. This is the perfect time to look back on how we have been doing education and research training, and discern what works while developing innovative approaches to improve on our successes. The maturity of 'nano' doesn't mean that this is the beginning of the end (as some no doubt are concerned, given the fashion-influenced nature of funding), but pedagogically, this is the end of the beginning.

DOUG NATELSON is a professor and a blogger based in the Department of Physics and Astronomy, Rice University, 6100 Main Street, Houston, Texas 77005, USA. e-mail: natelson@rice.edu 